\title{
Three-quadrant mucosal excision for solitary rectal ulcer syndrome
}

\author{
A. Privitera • T. C. Smyrk • E. J. Dozois
}

Received: 22 October 2009/Accepted: 17 December 2009/Published online: 4 February 2010

(C) Springer-Verlag 2010

\begin{abstract}
A 77-year-old female presented with a diagnosis of prolapsing hemorrhoids associated with rectal bleeding, a palpable mass and anal discomfort. Three-quadrant hemorrhoidal prolapse was noted on physical exam with an associated polypoid mass (Fig. 1). Lower endoscopy was otherwise negative. Three-quadrant mucosal excision was performed in the prone position to incorporate the mass and scattered satellite nodules. (Fig. 2). The wounds were reapproximated with absorbable sutures (Fig. 3). Postoperative recovery was uneventful. Histology confirmed solitary rectal ulcer (Fig. 4).
\end{abstract}

\footnotetext{
A. Privitera · E. J. Dozois $(\bowtie)$

Department of Surgery, Division of Colon and Rectal Surgery, Mayo Clinic, 200 First Street, SW, Rochester, MN 55905, USA e-mail: dozois.eric@mayo.edu

\section{A. Privitera}

e-mail: privitera@hotmail.com

\section{T. C. Smyrk}

Department of Pathology, Mayo Clinic College of Medicine, Rochester, MN, USA
}

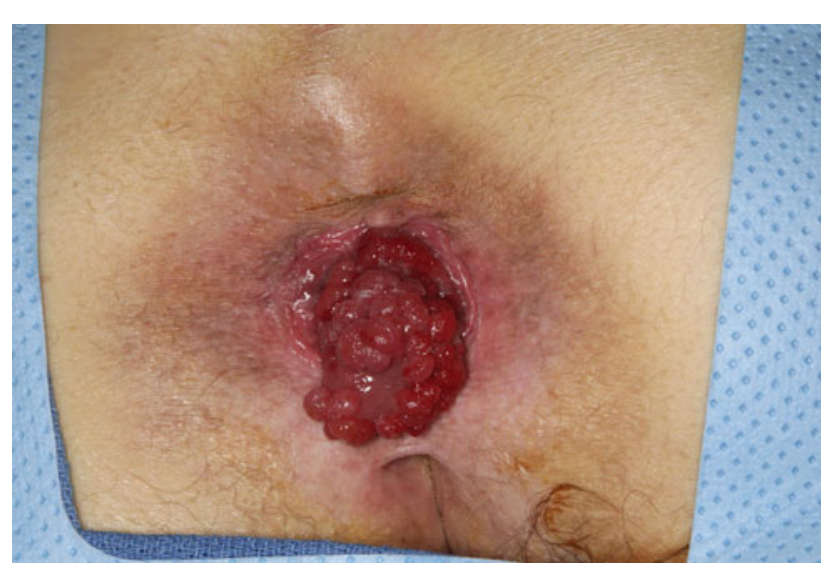

Fig. 1 Polypoid form of solitary rectal ulcer associated with mucosal prolapse

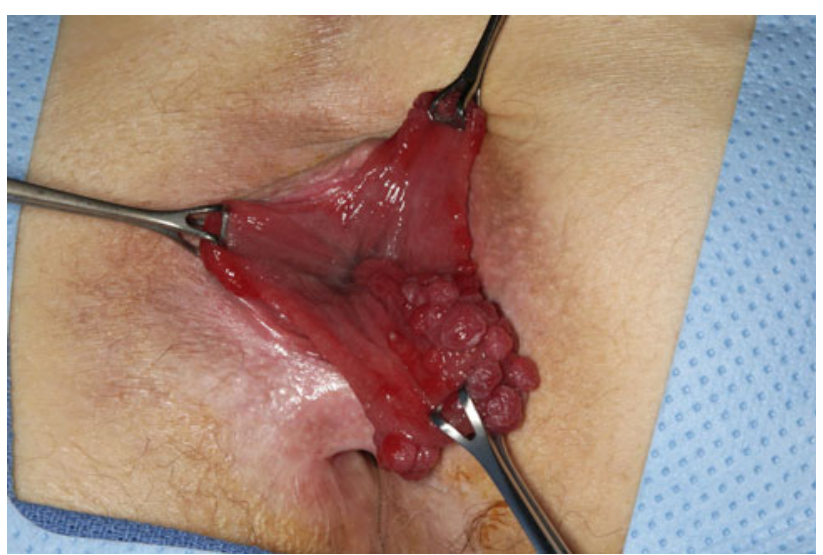

Fig. 2 Intraoperative findings: multiple small polypoid lesions noted 

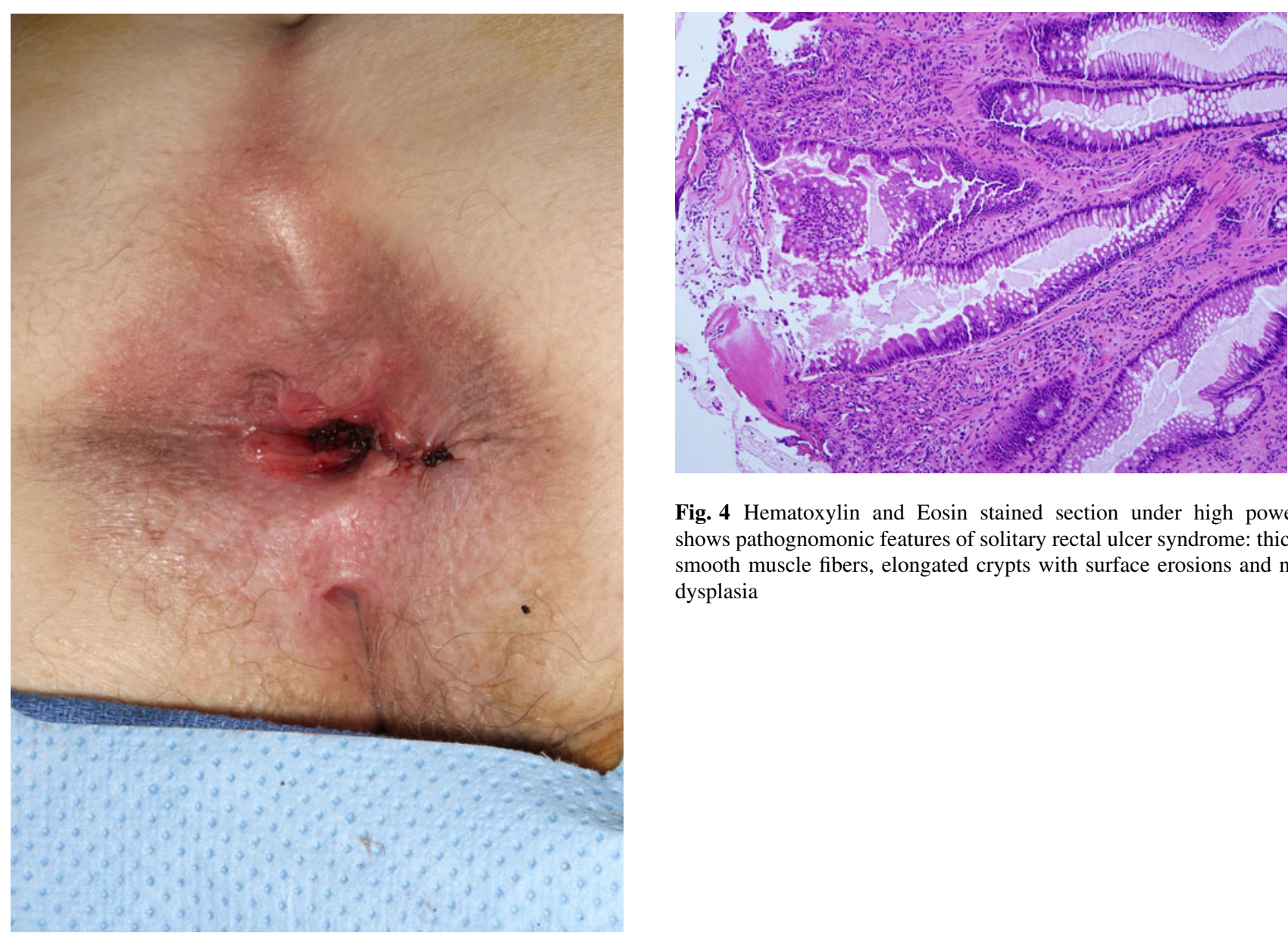

Fig. 4 Hematoxylin and Eosin stained section under high power shows pathognomonic features of solitary rectal ulcer syndrome: thick smooth muscle fibers, elongated crypts with surface erosions and no dysplasia

Fig. 3 Surgical excision with primary closure of mucosal defects 